\title{
Le motif « vieille » dans la toponymie de l'aire celtique
}

The 'hag' motif in the toponymy of the Celtic area

Lee Fossard

\section{OpenEdition}

\section{Journals}

Édition électronique

URL : https://journals.openedition.org/lbl/309

DOI : $10.4000 / \mathrm{lbl} .309$

ISSN : 2727-9383

Éditeur

Université de Bretagne Occidentale - UBO

\section{Édition imprimée}

Date de publication : 1 mai 2017

Pagination : 63-83

ISBN : 979-10-92331-31-8

ISSN : $1270-2412$

\section{Référence électronique}

Lee Fossard, "Le motif « vieille » dans la toponymie de l'aire celtique », La Bretagne Linguistique [En ligne], 21 | 2017, mis en ligne le 01 mai 2020, consulté le 25 mars 2022. URL : http:// journals.openedition.org/lbl/309; DOl : https://doi.org/10.4000/lbl.309

\section{(C) (1)}

La Bretagne Linguistique est mise à disposition selon les termes de la Licence Creative Commons Attribution 4.0 International. 


\title{
Le motif « vieille " dans la toponymie de l'aire celtique
}

\begin{abstract}
A
travers l'étude du motif « vieille», cet article vise à mettre en avant d'une part, des concordances entre des aires culturelles et linguistiques différentes ${ }^{1}$ et d'autre part, une méthode spécifique de recherche et de traitement de données ethnolinguistiques. Cette méthode est née d'un double constat. Premièrement, les études toponymiques concernant des personnages historiques, mythologiques ou légendaires sont rares $^{2}$, tandis que celles attribuées aux saints et à tout autre personnage chrétien (hagio-toponymie) sont abondantes. Deuxièmement, le type toponymique « vieille » est omniprésent sur les cartes anciennes (XIX ${ }^{\mathrm{e}}$ siècle) et modernes (XXe siècle) de la France et des îles Britanniques. Il se décline dans les différentes langues des aires linguistiques étudiées sous les variantes suivantes: Grac'h, Groac'h, Wrac'h, Gwrac'h, Vroac'h, Vrac'h, Groah ou Grah pour le breton ; Gwrach, Wrach pour le gallois ; Cailleach, Caillich pour le gaélique écossais et l'irlandais ; Calliagh, Cally ou Callee ${ }^{3}$ pour l'irlandais ; Carling pour l'écossais ; Old Woman, Old Wife, $\mathrm{Hag}^{4}$ pour l'anglais.
\end{abstract}

* Doctorant en Sciences du langage, CRBC/UBO (EA 4451), lee.fossard@gmail.com

1. Nous ferons quelques incursions dans les aires germanique et romane.

2. Cf. notamment, à titre d'exemples, les ouvrages de John Stuart STUART-GLENNIE (1869), André Soutou (1954, 1969), Jacques E. MERCERON (2006), Jacques LaCroiX (2007) et Stéphane GENDRON (2013).

3. Ces deux dernières variantes sont des formes anglicisées de Caillich.

4. Le mot hag provient du moyen anglais hagge et signifie avant tout : "An ugly old woman; [...]», «Une vieille femme laide; [...]» (Middle English Dictionary, s.v. hagge). Le 
Afin de rendre compte et d'expliquer la récurrence de ce motif, il est nécessaire d'adopter une approche pluridisciplinaire et de concevoir, en parallèle, une base de données pour répertorier et organiser les éléments issus des diverses sources bibliographiques consultées. En effet, on verra que le motif « (la) vieille ${ }^{5}$ » ne se limite pas à la toponymie : il concerne également les croyances (littérature orale, traditions, coutumes, etc.), la sémantique (zoonymes, phytonymes, météoronymes mais aussi expressions et dictons) et le mégalithisme (pierres dressées, tombes préhistoriques, dolmens, etc.).

D'un point de vue toponymique, il s'agit d'insister davantage sur l'aspect «commémoratif » que descriptif des noms de lieux. Cette toponymie commémorative, illustrée au niveau national par Stéphane Gendron (2013), est le témoin d'un ancrage de ces personnages (tels que Gargantua, Merlin, ou encore César) dans la culture et les mentalités des peuples. Envisagé sous un aspect diachronique ou synchronique, ce type d'étude peut nous permettre de savoir à quel point ces personnages sont connus dans les campagnes mais aussi comment les autochtones se les représentent.

Par l'étude du motif "vieille», on tentera de comprendre pourquoi les locuteurs d'une communauté linguistique ont attribué à cette figure : des montagnes, des vallées, des cours d'eau, des voies de communication ou encore des mégalithes. Pour ce qui est de la sémantique, on s'interrogera sur ce qu'implique le fait de nommer « vieille » des animaux, des plantes et des phénomènes atmosphériques. Enfin, on verra ce qu'apportent à notre réflexion les récits oraux associés à ce motif.

C'est donc à travers une approche pluridisciplinaire - toponymie, littérature orale et sémantique - que cet article se propose de rendre compte du motif "vieille ", principalement dans l'aire et la périphérie des parlers celtiques. On tentera par là-même de démontrer l'intérêt scientifique et l'aspect novateur des perspectives de recherches suscitées par une telle approche.

dictionnaire en ligne d'anglais moderne Merriam-Webster donne également, pour le même mot, le sens originel suivant : «An ugly, slatternly, or evil-looking old woman », "Une vieille femme laide, malpropre ou méchante» (Merriam-Webster, s.v. hag).

5. Il est très important de préciser que le motif « vieille » à l'étude est un substantif et non un adjectif. 


\section{La toponymie}

Science issue de l'onomastique, elle-même branche de la linguistique, l'étude des noms de lieux est par définition interdisciplinaire. Elle utilise et s'approprie des données d'autres disciplines - telles que l'histoire, l'archéologie ou la littérature orale - et, par ce biais, permet de contribuer à leur développement. Étudier les noms de lieux est une tâche complexe consistant à analyser leur signification, leur étymologie, leur évolution et l'influence qu'ils ont - ou ont eu - sur les sociétés humaines : nommer un lieu c'est se l'approprier, « en faire du territoire ${ }^{6}{ }$.

D’une manière générale, les noms de lieux - ou toponymes - possèdent deux fonctions principales. Lorsque des toponymes sont attribués à des personnages (historiques, religieux, légendaires, mythologiques ou simplement locaux), ils ont une fonction commémorative en ce sens qu'ils leur rendent hommage (en Bretagne, par exemple, on trouve de nombreux Liors Mam Goz, le « Jardin de la Grand-Mère »). Lorsqu'ils sont attribués à des éléments du paysage (végétation, relief, cours d'eau, voies de communication, etc.), ils ont une fonction descriptive (Gwaremm et Lann sont des toponymes bretons qui désignent une garenne et une lande).

Ces deux fonctions peuvent évidemment se combiner - on le voit bien dans l'exemple Liors Mam Goz - et parfois même, prendre un caractère métaphorique. Ainsi, dans le nord de l'Angleterre, de nombreuses collines rocheuses sont appelées King's Seat, le « Siège du Roi ». En Irlande, l'endroit appelé Calliagh Dirra's House ${ }^{7}$, c'est-à-dire la «Maison de la Vieille de Berre », n'est pas une habitation, comme on pourrait le penser, mais une allée couverte dédiée à un personnage mythologique.

Il faut également noter que certains toponymes, ne font pas référence à une réalité correspondant à la nature du lieu nommé : ils désignent en fait une réalité située au-delà de ce lieu même. Par exemple, le nom de lieu occitan Azuel ( Horizon $\left.^{9} »\right)$ signifie qu'à cet endroit

6. Salih AKIN (1999 : 9).

7. En Irlande, province de Leinster, comté de Louth, près de Monasterboice.

8. Toponyme recueilli dans le cadastre napoléonien du département de la Lozère.

9. Dictionnaire bilingue occitan-français en ligne (http:/ / www.panoccitan.org/diccionari. aspx?diccion $=\&$ lenga $=$ fr) s.v. asuèlh . 
le promeneur ou l'enquêteur pourra profiter d'un joli point de vue sur le paysage alentour. On note ici qu'un nom de lieu peut donc faire référence à un ou plusieurs repères terrestres éloignés de lui. On verra que ce principe est primordial dans l'explication du type « vieille » en toponymie.

À la lumière des trois exemples précédents, il est nécessaire de mener des enquêtes de terrain ou, tout du moins, de se rendre sur place afin de vérifier ses propres hypothèses. À défaut, l'enquêteur peut s'en remettre à la consultation de cartes très précises permettant d'observer le relief et/ou la nature du terrain via des prises de vues par satellites.

\section{La méthodologie adoptée dans l'étude du motif " vieille »}

Nous allons décrire succinctement la méthode adoptée. Elle se divise en deux temps : la recherche et le traitement des données.

\section{La recherche des données : les sources écrites}

Les documents indiqués ci-dessous sont donnés à titre d'exemples et sont disponibles sur Internet, dans les bibliothèques municipales ou aux Archives départementales. J'invite le lecteur intéressé par ces sources, et souhaitant plus de détails, à consulter la première partie de l'ouvrage de Stéphane Gendron $\left(2008^{10}\right)$.

1. Sources administratives et historiques

Certaines d'entre elles permettent de retrouver les plus anciennes attestations d'un toponyme. Les documents principalement utilisés sont, par ordre chronologique :

- les œuvres de littérature antique comme celles de Ptolémée, Xénophon ou Homère.

- les cartulaires (datant du Moyen Âge), terriers et compoix (du

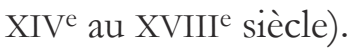

- le cadastre napoléonien (début XIX ${ }^{\mathrm{e}}$ siècle) : à la différence du cadastre moderne, il comprend les noms des parcelles de terrain (microtoponymes) de chaque commune de France métropolitaine. Il est composé de documents appelés États de Sections com- 
portant des données détaillées sur les terrains recensés (superficie, type de terrain, etc.). Chaque parcelle est ensuite numérotée et représentée sur un plan cadastral. Les gazetteers - équivalents britanniques du cadastre napoléonien - ont un fonctionnement quasi identique mais leur consultation en ligne est payante.

- les dictionnaires topographiques (seconde moitié du XIX ${ }^{\mathrm{e}}$ siècle, réédités dans les années 1980-1990).

- les bases de données toponymiques régionales ou nationales comme la base FANTOIR ${ }^{11}$ : celle-ci répertorie tous les noms des voies et lieux-dits de tous les départements français et comprend plus de 7,5 millions de toponymes.

\section{Sources scientifiques}

Toute étude toponymique, couplée à une étude sémantique, doit être complétée et affinée via des ouvrages de référence en linguistique, notamment :

- les dictionnaires anciens ou récents des langues celtiques.

- les dictionnaires toponymiques et/ou étymologiques tels que les ouvrages d'Albert Dauzat et Charles Rostaing (1963), d'Ernest Nègre $\left(T G F^{12}\right)$; de Walther von Wartburg $\left(F E W^{13}\right)$; etc.

- les glossaires dialectaux comme celui d'André Pégorier ${ }^{14}$.

- les atlas linguistiques régionaux, nationaux ou européens ( $A L E$ Atlas Linguarum Europae).

- les travaux de toponymie descriptive, outils importants pour mieux comprendre la toponymie locale : on pense par exemple aux ouvrages de Patrick Weston Joyce (1869, 1871 et 1913) pour l'Irlande, William John Watson (1904) pour l'Écosse, Thomas Morgan (1912) pour le pays de Galles, Albert Dauzat (1939) pour la France, François Falc'hun (1966), Bernard Tanguy (1975) et Pierre Hollocou \& Jean-Yves Plourin (2014) pour la Bretagne.

11. La base FANTOIR (Fichier ANnuaire TOpographique Initialisé Réduit), anciennement appelée base RIVOLI (Répertoire Informatisé des VOies et Lieux-dits) a été établie par le ministère des Finances.

12. E. NĖGRE, Toponymie Générale de la France, Genève, Droz, 1990-1991.

13. W. von WARTBURG, Franæösisches Etymologisches Wörterbuch, Leipzig, Tübingen, Bâle, 1922-1987.

14. A. PÉGORIER, Les noms de lieux en France : glossaire des termes dialectaux, Paris, Institut Géographique National, 1997. 
Les mentions toponymiques de personnages magico-religieux (fées, géant(e)s, sorcières, etc.) conduisent le chercheur à consulter également des ouvrages sur :

- la littérature orale, comme ceux de Donald Alexander Mackenzie (1917) et d'Eleanor Hull (1927) pour l'Écosse et l'Irlande ; d'Arnold van Gennep (1947) pour la France ; de Nicole Belmont (1986) pour ses travaux d'ethnologie à l'échelle européenne ;

- les traditions et coutumes, comme ceux de Paul Sébillot (1881) et Pierre Saintyves (1936) pour la France; Gerhard Rohlfs (1966) et James George Frazer (1983) pour une approche interculturelle ; etc.

- l'archéologie, comme celui de William Copeland Borlase (1897) sur les mégalithes d'Irlande. On consultera également les sites internet collaboratifs tels que The Modern Antiquarian de Julian Cope ${ }^{15}$, The Megalithic Portal d'Andy Burnham ${ }^{16}$, ou encore le site de la Royal Commission on the Ancient and Historical Monuments of Scotland ${ }^{17}$ (RCAHMS). Ces sites fournissent des renseignements non négligeables sur la situation géographique, la description précise et le nom des mégalithes.

\section{Cartes topographiques}

Elles sont à la base de la recherche toponymique car elles permettent d'étudier un nom de lieu de manière diachronique ou diatopique. Dans un premier temps, il est nécessaire de consulter les registres manuscrits et/ou d'interroger les barres de recherche en ligne en quête du toponyme "vieille » dans la langue de la zone étudiée. Dans un second temps, si cela est nécessaire, il s'agit de parcourir du regard les cartes en question : celles de ScotlandsPlaces pour l'Écosse, d'Ordnance Survey Ireland pour l'Irlande (remontant jusqu'au milieu du

15. J. Cope, The Modern Antiquarian, 2000-2016, (http://www.themodernantiquarian. com/home). Sites mégalithiques du Royaume-Uni, d'Irlande et d'Europe.

16. A. Burnham, The Megalithic Portal, 1997-2016, (http://www.megalithic.co.uk/index. php). Sites préhistoriques en Europe et dans le monde.

17. La commission royale des monuments historiques et préhistoriques d'Écosse, (https:// canmore.org.uk/). Site géré depuis le 1er octobre 2015 par un nouvel organisme appelé Historic Environment Scotland. 
XIX $^{\mathrm{e}}$ siècle $^{18}$ ); celles de Get a map, Ordnance Survey, OS OpenSpace ${ }^{19}$ pour le Royaume-Uni (datant du XXe siècle) et celles de l'IGN ${ }^{20}$ (parmi lesquelles figure la carte de Cassini du XVIII e siècle).

Les cartes contemporaines de Google Maps offrent un bon aperçu de la topographie des lieux, notamment par le biais de Google Street View. Cet outil, permettant de se projeter sur place et d'observer un lieu à partir d'une voie de communication, peut révéler de nouveaux toponymes sur des panneaux de signalisation. Ainsi, le panneau indiquant le nom d'un village irlandais appelé Kishkeam (comté de Cork) nous informe que ce toponyme est une forme anglicisée. En effet, sur ce même panneau figure son équivalent irlandais : Coisceim na Cailli, c'est-à-dire le « $\operatorname{Pas}^{21}$ de la Vieille », toponyme quasiment absent des cartes d'état-major.

\section{La recherche des données : les sources orales}

Après avoir effectué ce travail de recherches bibliographiques, il est intéressant de mener des enquêtes de terrain qui peuvent s'avérer très utiles pour expliquer la signification d'un nom de lieu. L'enquête se déroule en 3 étapes :

1. Repérage du lieu étudié à l'aide des deux matériaux suivants

- les photocopies des différentes cartes - modernes (avec vue par satellites) et anciennes - sur lesquelles figure le toponyme étudié et sur lesquelles on inscrit ses coordonnées géographiques.

- un GPS possédant la fonction de recherche d'un lieu par coordonnées.

2. Rencontre avec les locaux

L'enquêteur montre à l'informateur les photocopies des cartes et lui pose les questions suivantes en faisant bien attention de ne pas

18. http://maps.osi.ie/publicviewer pour l'Irlande et http://www.scotlandsplaces.gov. uk/ pour l'Écosse. Les cartes de la Bibliothèque nationale d'Écosse datent pour les plus anciennes du XVIe s. (http://maps.nls.uk/index.html).

19. https://www.ordnancesurvey.co.uk/osmaps ou http://www.explorebritain.info/ search

20. http://www.geoportail.gouv.fr/accueil

21. Cf. le dictionnaire bilingue anglais-irlandais (http://www.tearma.ie/Home.aspx) s.v. coiscéim et s.v. step. 
l'influencer : "Savez-vous que cet endroit s'appelle La Colline de la Vieille? », «Savez-vous pourquoi cet endroit s'appelle ainsi ? », «Y a-t-il d'autres endroits de ce type dans la région ?", etc.

3. Recueil des informations

La plupart du temps, cette collecte prend la forme de notes écrites in situ ou, après avoir obtenu l'accord de l'informateur, d'enregistrements audio à l'aide d'un dictaphone ou d'un téléphone portable.

\section{Le traitement des données ${ }^{22}$ : la base de données}

Elle se présente sous la forme d'un tableur ${ }^{23}$ permettant de classer et d'exploiter les données d'un personnage magico-religieux repérées parmi les différentes sources énoncées précédemment. Le tableur comporte autant de lignes que d'éléments et neuf colonnes pour neuf critères de classification. Ces critères sont les suivants :

Colonne A (MATRI.) : chaque élément de la base possède un matricule basé sur la zone géographique dans laquelle il se trouve (cf. tableau ci-dessous) :

\begin{tabular}{|l|l|}
\hline Matricules & \multicolumn{1}{|c|}{ Zones géographiques } \\
\hline FRB22 & FRance - Bretagne - Côtes-d'Armor (22) \\
\hline FRB29 & FRance - Bretagne - Finistère (29) \\
\hline FRB35 & FRance - Bretagne - Ille-et-Vilaine (35) \\
\hline FRB56 & FRance - Bretagne - Morbihan (56) \\
\hline ECNO & ÉCosse du Nord-Ouest \\
\hline ECSE & ÉCosse du Sud-Est \\
\hline GALL & pays de GALLes \\
\hline IRLC & IRLande - Connaught \\
\hline IRLL & IRLande - Leinster \\
\hline IRLM & IRLande - Munster \\
\hline IRLU & IRLande - Ulster \\
\hline
\end{tabular}

Exemple : le matricule IRLU069 correspond au 69e élément repéré en Irlande dans la province d'Ulster.

22. S. GENDRON (2013 : 17) nous rappelle que « l'intelligence et la compréhension des lieux passent par le catalogage, la cartographie, l'étude des langues et le recueil des traditions locales $»$.

23. Établi via Microsoft Office ou Open Office. 
Colonne B (NOM EXACT) : nom de l'élément tel qu'il a été trouvé dans la source bibliographique. Il s'agit majoritairement du nom d'un lieu mais il peut également s'agir d'une légende, d'une expression, d'un zoonyme, etc.

Colonne C (TRAD.) : traduction (ou proposition de traduction) de l'élément en français.

Colonne D (LOCA. 1) : localisation « élargie » de l'élément (région, province, etc.).

Colonne E (LOCA. 2) : noms des villes ou villages les plus proches de l'élément.

Colonne $\mathbf{F}$ (COORD.) : coordonnées géographiques repérées entre autres grâce à Google Earth ${ }^{24}$. Un astérisque indique une localisation approximative.

Colonne G (TYPE) : type de l'élément (légende, tradition, etc.) ou réalité désignée (chemin, gué, village, mégalithe, insecte, etc.).

Colonne H (COMM.) : commentaires divers et variés sur l'élément (observations, citations et références bibliographiques, formes anciennes attestées, etc.).

Colonne I (BIBLIO.) : références bibliographiques abrégées.

L'avantage de cette base de données, en constante évolution, est son côté pratique et malléable. On peut appliquer des tris ou des filtres aux éléments enregistrés : par exemple, faire apparaître uniquement les puits ou les hauteurs attribuées à un personnage magico-religieux pour établir des statistiques à son sujet.

\section{Le traitement des données : la cartographie}

Afin d'avoir une vue d'ensemble et d'analyser sur le plan diatopique les noms de lieux répertoriés dans cette base, il est nécessaire de les cartographier. Pour cela, sur une carte personnelle enregistrée sur Google Maps ou Google Earth, on positionne à l'aide d'épingles virtuelles les toponymes en fonction de leurs coordonnées géographiques ou en fonction des renseignements donnés par les différentes sources bibliographiques. 
À titre d'exemples, voici 10 éléments extraits de la base de données et classés selon les 6 premières colonnes ${ }^{25}$ :

\begin{tabular}{|c|c|c|c|c|c|}
\hline A & B & C & D & E & $\mathbf{F}$ \\
\hline MATRI. & NOM EXACT & TRAD. & LOCA. 1 & LOCA. 2 & COORD. \\
\hline IRLU069 & $\begin{array}{l}\text { Shanven ou The Old } \\
\text { Woman (or Hag) }\end{array}$ & La Vieille & Ulster - Moyle & Cushendun - Altagore & $\begin{array}{l}550859 \mathrm{~N}- \\
060259 \mathrm{~W}^{*}\end{array}$ \\
\hline IRLC034 & Gubnagalliagh & Pointe de la Vieille & Connacht - Mayo & Dooghbeg & $\begin{array}{l}535231 \mathrm{~N}- \\
094917 \mathrm{~W}\end{array}$ \\
\hline GALL084 & Cwm Pwll y Wrach & $\begin{array}{l}\text { Vallée du Bassin } \\
\text { de la Vieille }\end{array}$ & Powys & Talgarth & $\begin{array}{l}515909 \mathrm{~N}- \\
031248 \mathrm{~W}\end{array}$ \\
\hline GALL053 & Ffynnon y Wrâch & Puits de la Vieille & Isle of Anglesey & Holyhead Mountain & $\begin{array}{l}531827 \mathrm{~N}- \\
044005 \mathrm{~W}\end{array}$ \\
\hline FRB3501 & $\begin{array}{l}\text { La Vieille et la Pierre } \\
\text { Droite }\end{array}$ & & $\begin{array}{l}\text { Ille-et-Vilaine - } \\
\text { Brocéliande }\end{array}$ & $\begin{array}{l}\text { Maure-de-Bretagne - } \\
\text { Loutehel - Pierre Droite }\end{array}$ & $\begin{array}{l}475652 \mathrm{~N}- \\
020358 \mathrm{~W}\end{array}$ \\
\hline FRB2929 & Coc'h ar Groac'h & Étron de la Vieille & Finistère & $\begin{array}{l}\text { Penmarc'h - Phare } \\
\text { d'Eckmuhl }\end{array}$ & $\begin{array}{l}474805 \mathrm{~N}- \\
042336 \mathrm{~W}\end{array}$ \\
\hline FRB2215 & La Pierre de la Vieille & & Côtes-d'Armor & Plourac'h & $\begin{array}{l}482459 \mathrm{~N}- \\
033251 \mathrm{~W}^{*}\end{array}$ \\
\hline ECSE164 & $\begin{array}{l}\text { Cailleach - The Corn } \\
\text { Mother }\end{array}$ & $\begin{array}{l}\text { La Vieille - La } \\
\text { Mère du blé }\end{array}$ & Perthshire & $\begin{array}{l}\text { Strath Fillan - } \\
\text { Crianlarich }\end{array}$ & $\mathrm{N} / \mathrm{A}$ \\
\hline ECSE188 & Carlin Tooth & Dent de la Vieille & $\begin{array}{l}\text { Scottish Borders - } \\
\text { Hawick }\end{array}$ & Southdean & $\begin{array}{l}551853 \mathrm{~N}- \\
023455 \mathrm{~W}\end{array}$ \\
\hline ECNO162 & Ob na Caillich & Baie de la Vieille & $\begin{array}{l}\text { Highlands - Ross } \\
\text { \& Cromarty }\end{array}$ & $\begin{array}{l}\text { Applecross - Annat - } \\
\text { Torridon? }\end{array}$ & indéterminée \\
\hline
\end{tabular}

\section{Quelques résultats et analyses}

Dans les parties suivantes, nous allons exposer des résultats statistiques et cartographiques, puis nous étudierons brièvement des données issues de la littérature orale et de la sémantique afin de mieux comprendre ce que revêt le motif «vieille».

\section{En toponymie ${ }^{26}$}

Le tableau ci-dessous donne des statistiques non exhaustives (année 2015) aussi bien dans l'aire linguistique celtique que dans les aires germanique et romane :

25. Pour des questions de lisibilité, seules 6 colonnes sur 9 sont affichées et la traduction des éléments ne figure pas entre guillemets.

26. Les toponymes qui suivent ont principalement été répertoriés à partir des cartes d'étatmajor (citées précédemment) des pays correspondants, mais aussi à partir de la base FANTOIR et du cadastre napoléonien pour la France. 


\begin{tabular}{|l|l|}
\hline Pays & Toponymes composés du motif « la vieille » \\
\hline \multirow{3}{*}{ Îles Britanniques } & $\begin{array}{l}>940 \\
(470 \text { en Écosse, } 260 \text { en Irlande, } 90 \text { au pays de Galles } \\
\text { et } 120 \text { en Angleterre })\end{array}$ \\
\hline \multirow{3}{*}{ France } & $\begin{array}{l}>800 \\
\text { (dont environ } 200 \text { dans la région Bretagne et } \\
80 \text { dans le département de la Lozère) }\end{array}$ \\
\hline Norvège & $>600$ \\
\hline Espagne & $>430$ \\
\hline Portugal & $>200$ \\
\hline
\end{tabular}

À titre d'exemples, en Bretagne, on trouve les toponymes: Kergwrac'b « le Village ou la Maison de la Vieille », Porz-Grac'b « Port de la Vieille », Guernégronac’h « Marais de la Vieille » mais aussi Mener-Grah «Pierre de la Vieille » ou encore Bez ar Wrac'b « Tombeau de la Vieille». Ces deux derniers désignent généralement des mégalithes. Un des plus grands menhirs d'Europe de l'Ouest - si ce n'est le plus grand ${ }^{27}-$ est situé près de Locmariaquer dans le Morbihan, il est appelé Men-erGroach : la « Pierre de la Vieille».

Au pays de Galles, il existe près de Capelulo (comté de Conwy), une concentration de menhirs et de cromlechs située non loin d'une grande carrière datant du néolithique et d'un petit lac appelé Llyn y Wrach, le «Lac de la Vieille» (Google Maps; OS OpenSpace). À l'horizon de ce lac et d'un des cercles de pierres ${ }^{28}$, se dessine l'étrange silhouette ventrue d'une colline nommée Foel Lûs (cf. photo de l'auteur ci-dessous).

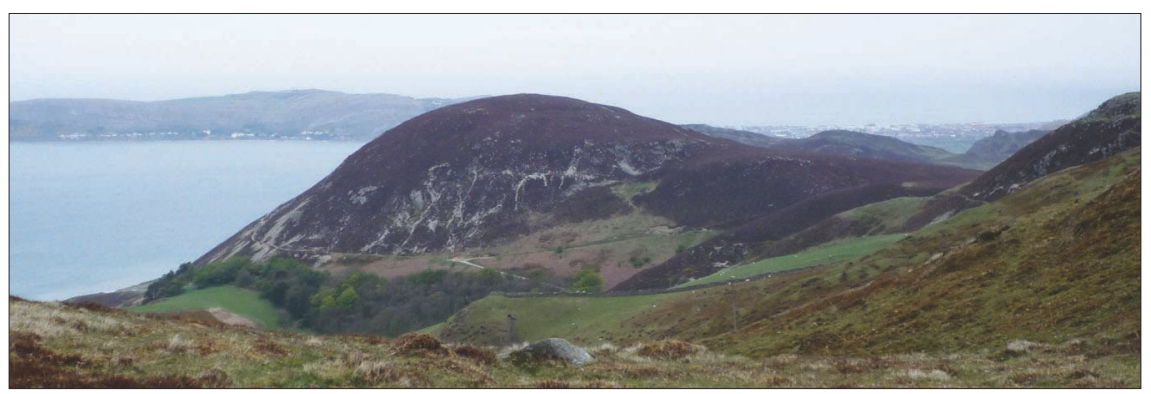

Le Foel Lûs, comté de Conwy, Capelulo (pays de Galles).

27. Menhir brisé en quatre morceaux, mesurant près de 20 mètres.

28. D'environ 20 mètres de diamètre. 
En Angleterre, au sud de Whitby, près de Stape (North Yorkshire), se trouve un puits appelé Old Wives Well, le « Puits des Vieilles ou de la Vieille ${ }^{29} \gg$ (OS OpenSpace). À l'ouest de ce puits, à 3 kilomètres à vol d'oiseau, on trouve également un tumulus appelé Old wife's round mound barrow, le «tumulus rond de la Vieille » (Ancient Monument ${ }^{30}$ ).

En Écosse, près de Craighouse (île de Jura), 4 toponymes sont regroupés en bord de mer : A' Chailleach, «la Vieille», Iochdar na Caillich, « au Pied de la Vieille ${ }^{31} »$, Rubha na Caillich, le "Promontoire de la Vieille» et Port na Caillich, le «Port de la Vieille». On constate que, du haut du « Promontoire de la Vieille », on aperçoit parfaitement les fameuses collines de l'île de Jura appelées Paps of Jura: les "Seins de Jura ${ }^{32} »$ (cf. photo de l'auteur ci-dessous). Lorsqu'on descend de ce promontoire, on ne les voit plus.

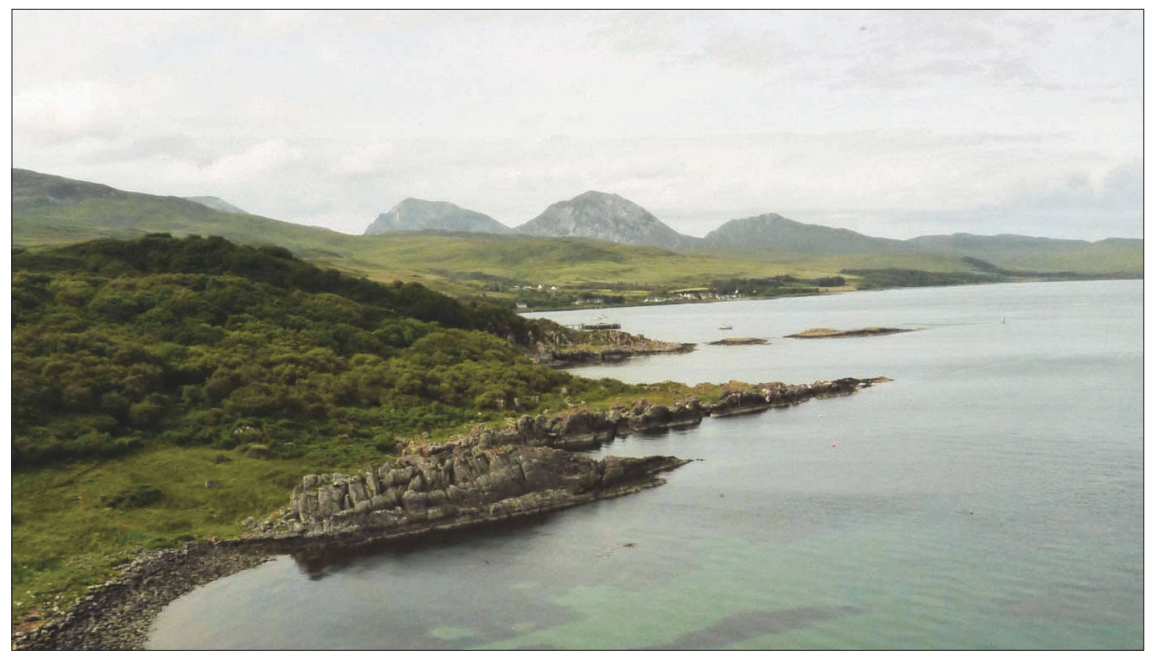

Les « Seins de Jura » visibles du « Promontoire de la Vieille » (Écosse, île de Jura).

29. D’un point de vue phonétique, il y a très peu de différences entre Old Wife's Well et Old Wives Well.

30. http://www.ancientmonuments.info. Site collaboratif répertoriant les monuments (pré)historiques de Grande-Bretagne.

31. Le dictionnaire bilingue anglais-gaélique écossais Dwelly (inclus dans Am Faclair Beag) indique au mot iochdar « The bottom, lowest part [...]», « Le bas, la partie la plus basse [...]». Ainsi, on pourrait aussi traduire ce toponyme par « en Bas de la Vieille».

32. http://www.dsl.ac.uk/entry/dost/pap_n_1. Dictionnaire bilingue écossais-anglais Dictionary of the Scots Language (DSL) s.v. pap. 
En Irlande, dans le comté de Kerry, le Carrauntoobil - plus haute montagne du pays (1039 mètres) - domine un cirque géologique appelé Com Caillí ou Hag's Glen (Ordnance Survey Ireland) c'est-à-dire le "Cirque ${ }^{33}$ de la Vieille » ou la «Vallée de la Vieille». Deux protubérances rocheuses se dessinent sur un des flancs de cette vallée, elles sont appelées Hag's Teeth (small) et Hag's Teeth (large), c'est-à-dire les «Dents de la Vieille (petites)» et les « Dents de la Vieille (grandes)» (Ordnance Survey Ireland). Sur la même carte, on dénombre également trois autres endroits comportant le motif «vieille»: une rivière appelée Tooreenteecallee située en amont d'un lac appelé Loch Caillí - anglicisé en Lough Calleeet, au milieu de ce lac, un îlot nommé Crompannnacallee.

Voici, en résumé, un aperçu de la répartition géographique des noms de lieux de type «vieille» pour les zones étudiées précédemment (carte faite par l'auteur en 2014) :

Toponymes composés du motif « la vieille » dans les îles Britanniques.

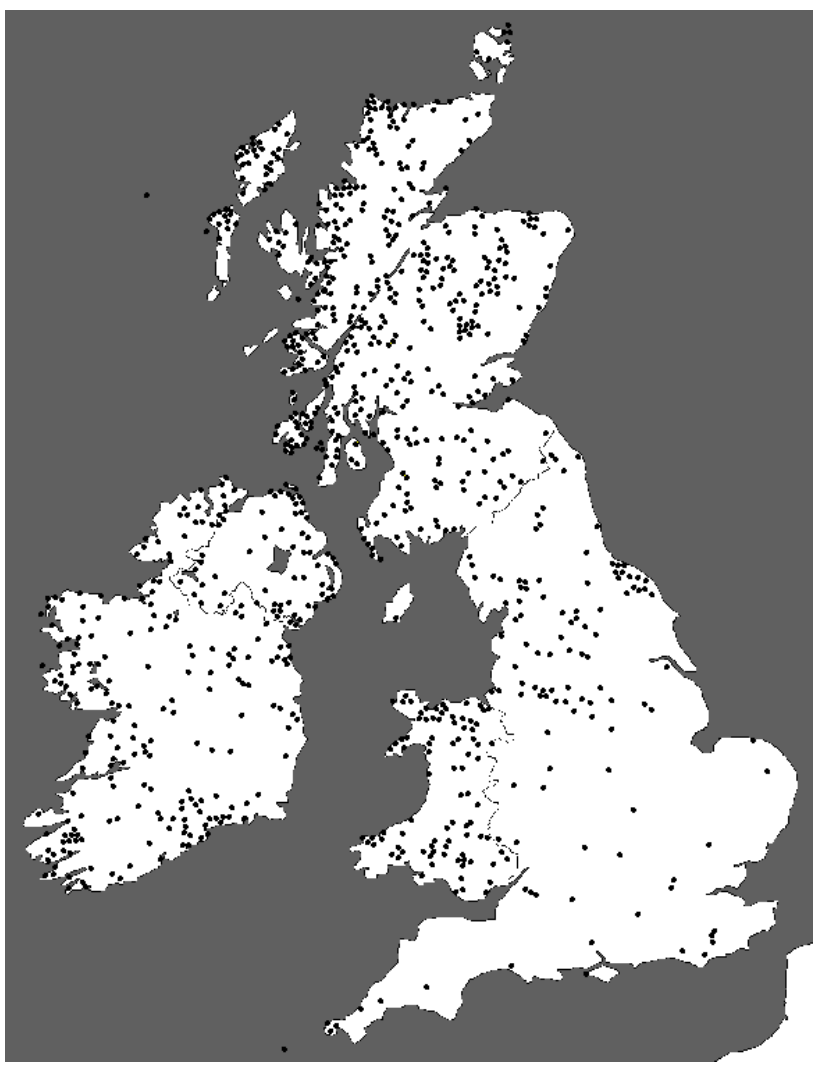

33. Dictionnaire bilingue anglais-irlandais Téarma.ie s.v. com. 


\section{En littérature orale}

Le principe est de savoir si des légendes existent autour d'un lieu comportant le motif «vieille» et si elles peuvent fournir des informations intéressantes quant à la description de ce personnage.

Dans les mythes et légendes, la Vieille est parfois connue sous le nom de Cailleach Beinn-a-Bhric (Alexander Robert Forbes, 1905 : 129) ou Beira (Mackenzie, 1917) en Écosse et Cailleach Bheara ${ }^{34}$ en Irlande (Hull, 1927). La première vit dans la montagne nommée Beinn a' $\mathrm{Bhric}^{35}$ et la dernière est originaire de la péninsule de Beara (sud-ouest de l'Irlande). En Écosse, elle est décrite sous la forme d'une géante façonnant le paysage avec son marteau (Mackenzie, 1917). Elle incarne également le cycle des saisons puisqu'elle devient vieille et laide en hiver et jeune et belle en été, son apparence se dégradant au fil des saisons (Mackenzie, 1917 : 24). Son châle est tellement grand qu'elle doit le laver dans la mer - plus précisément dans le Corryvreckan, un gigantesque tourbillon situé entre les îles de Scarba et de Jura - et le faire sécher sur les montagnes : il symbolise ainsi la neige (Mackenzie, $1917: 30-31)$.

La Vieille est également une géante bâtisseuse. Elle construit des montagnes (Little Wyvis, 764 mètres d'altitude) ou encore des îles (les Hébrides) en transportant des rochers et de la terre dans son panier de pêche ou son tablier (Hull, 1927). En Irlande, dans la zone mégalithique de Tara, à Loughcrew (comté de Meath), elle aurait involontairement créé un grand tumulus en laissant tomber des pierres de son tablier alors qu'elle sautait d'une colline à une autre (Hull, 1927 : 246). Ce tumulus - appelé Hag's Carn, le « Cairn de la Vieille »- est situé sur la plus haute colline du comté de Meath, elle-même appelée Slieve na Calliagh $^{36}$, la « Colline de la Vieille».

34. Avec différentes variantes pour la graphie du mot bheara.

35. Située dans les Highlands (Invernesshire), à l'est de Kinlochmore, près de Fort Williams.

36. Ou Slieve na Cailli (cf. Ordnance Survey Ireland). 
On retrouve en Bretagne, à Plouarzel (Finistère), la même caractéristique au sujet du grand menhir de $\operatorname{Kerloas}^{37}$ : une femme l'aurait apporté dans son tablier (Georges Guénin, 1910). Ce même mégalithe, pourvu de deux bosses à sa base, faisait l'objet d'une coutume : les nouveaux mariés venaient se frotter le ventre nu contre l'une de ses bosses (Chevalier de Fréminville, 1832). On peut peut-être y voir les traces d'anciens rites de fécondité.

En outre, D. A. Mackenzie ajoute au gigantisme de la Vieille Beira qu'elle était «the mother of all the gods and goddesses in Scotland ${ }^{38}$ » (1917: 22) mais aussi déesse de la nature, et tout particulièrement du climat, des rivières, des lacs et des montagnes (1917 : 16). On rencontre également ce lien entre la Vieille et le climat dans l'aire linguistique romane. En effet, dans les Asturies (nord de l'Espagne), près des montagnes appelées Picos de Europa, quand il commençait à neiger, les bergers avaient pour habitude d'interpeller la Vieille pour lui demander : «¿Qué traes, Vieya? », c'est-à-dire "Qu'apportes-tu, la Vieille ? " (Cristobo de Milio Carrín, 2008 : 18). En Sardaigne, par temps de sécheresse, lors de processions, les enfants imploraient la Vieille (tsia bezza en sarde) pour qu'elle apporte la pluie (Michel Contini, 2012 : 377).

\section{En sémantique}

Sur ce dernier point, nous allons présenter un aperçu des différents signifiés de l'unité lexicale «vieille » principalement dans les langues celtiques et romanes. J'invite les personnes intéressées par cet aspect à consulter le tableau plus complet - néanmoins non exhaustif - de mon mémoire de recherche en linguistique ${ }^{39}$.

37. Menhir mesurant environ 9 mètres de haut.

38. « la mère de tous les dieux et déesses d'Écosse ».

39. Lee FossarD, La Vieille dans la toponymie du Royaume-Uni et de l'Irlande : trace d'un ancien culte voué à la Nature?, Mémoire de master 2 recherche, spécialité linguistique, Université Stendhal-Grenoble 3 (Université Grenoble Alpes), 2014. Disponible en ligne à <dumas-01080422> (ou http://dumas.ccsd.cnrs.fr/dumas-01080422/document). Le tableau en question est en page 12. 


\begin{tabular}{|c|c|}
\hline \multicolumn{2}{|r|}{ Les désignations du signifiant « la vieille» } \\
\hline $\begin{array}{l}\text { Phénomènes } \\
\text { atmosphériques }\end{array}$ & $\begin{array}{l}\text { Arc-en-ciel, canicule, nuage, tonnerre et lune } \\
\text { (Mario Alinei \& Francesco Benozzo, } 2012: 69 \text { ) } \\
\text { Brouillard } \\
\quad \text { Italie du nord : province de Brescia (Rohlfs, } 1966: 116 \text { ) } \\
\text { Tempête } \\
\quad \text { Îles Canaries (Rohlfs, } 1966: 119 \text { ) } \\
\text { Soleil, neige } \\
\quad \text { Allemagne : Rhénanie (Rohlfs, } 1966: 116,117 \text { ) }\end{array}$ \\
\hline Éléments naturels & $\begin{array}{l}\text { Étincelle } \\
\quad \text { Italie : régions de Campanie et des Marches (Rohlfs, } 1966 \text { : } \\
\text { 130) ; Bretagne (Favereau, 1992, s.v. gwrac'henn) } \\
\text { Avalanche, éboulement et montagne } \\
\quad \text { (Benozzo, } 2011: 17 \text { ) }\end{array}$ \\
\hline Animaux & $\begin{array}{l}\text { Ours, belette, serpent, crapaud, luciole, courtilière, etc. } \\
\text { (Alinei \& Benozzo, } 2012: 69 \text { ) } \\
\text { Coucou, martinet, pinson des bois, hirondelle, etc. } \\
\text { pays de Galles (GPC, s.v. gwrach y cae, gwrach yr ellyll, gwrachan) } \\
\text { Roitelet } \\
\text { Corse (Muriel Poli, } 2015: 36-37 \text { ) } \\
\text { Cormoran huppé } \\
\text { Écosse (Dwelly-d, s.v. cormorant) } \\
\text { Poissons divers dont les labres } \\
\text { pays de Galles (Daniel S. Evans, } 1858: 936 \text {; GPC, } \\
\text { s.v. gwrach, gwrachan) ; France, Bretagne, pays de Galles, } \\
\text { Cornouailles (Le Bris \& Carpitelli, } 2013: 59-60^{40} \text { ); Corse } \\
\text { (Poli, } 2015: 36 \text { ) } \\
\text { Araignée, punaise } \\
\text { Italie : respectivement, région de Calabre, province de } \\
\text { Cosenza et région de Toscane, province de Pistoia (Rohlfs, } \\
\text { 1966: } 129 \text { ) } \\
\text { Cloporte, mille-pattes et puceron } \\
\text { pays de Galles (Evans, } 1858: 277,341,614 \text { ) ; Bretagne } \\
\text { (Favereau, 1992, s.v. gwrac'h) }\end{array}$ \\
\hline Plantes & $\begin{array}{l}\text { Gerbe de blé, andain et botte de foin } \\
\text { pays de Galles (GPC, s.v. gwrach, gwrachan) } \\
\text { Bardane, prêle et belladone } \\
\text { pays de Galles (GPC, s.v. cedowrach) }\end{array}$ \\
\hline
\end{tabular}

40. Daniel LE BRIS \& Elisabetta CARPITELLI, «Concordances aréales en zone Atlantique », dans E. Carrilho, C. Magro \& X. Alvarez (dir.), Current Approaches to Limits and Areas in Dialectology, Cambridge, Scholars Publishing, 2013, p. 49-68. 
D’une manière générale, on peut expliquer cette profusion de référents extra-linguistiques par deux arguments pouvant se combiner. Le premier argument tend à montrer qu'il existe des caractéristiques morphologiques et/ou comportementales communes à tous ces référents. On remarque cependant qu'il est parfois compliqué, voire périlleux, de trouver une telle ressemblance entre un oiseau, une plante et une avalanche. De plus, cela n'explique pas pourquoi on a affaire au terme « vieille » et non au terme « vieux » par exemple. Le deuxième argument permet de combler les lacunes du premier puisqu'il vise à démontrer, à la lumière des données recueillies en ethnologie, littérature orale ou mythologie, que ces référents sont les traces linguistiques de croyances en une divinité. On peut donc faire le lien avec deux caractéristiques divines de la Vieille présentées précédemment : déesse du climat et déesse de la nature. De plus, Mario Alinei et Francesco Benozzo nous informent que « ces dénominations identifient une ancêtre totémique matrilinéaire $[. .$.$] à l'origine dominatrice des animaux et de la nature$ $[\ldots] »(2012:$ 69).

Les deux auteurs précités (2012) rappellent que ces différents signifiés participent d'une vénération et/ou d'une sacralisation des animaux, plantes et phénomènes atmosphériques datant, pour les premiers, du Paléolithique (au moins 9000 avant J.-C.) et, pour les derniers, du Mésolithique final (environ 6000 avant J.-C.). On peut supposer que cette part de religieux du lexique est due à la fascination suscitée par les caractéristiques, les propriétés et l'apparence ${ }^{41}$ de ces référents extra-linguistiques. Les premiers peuples qui les ont nommés « vieille» l'ont certainement fait en référence à la divinité éponyme. Ces animaux, plantes et autres phénomènes naturels appelés ainsi sont autant de traces ethnolinguistiques des liens étroits entre l'Homme et la Nature.

$$
*
$$

41. L'arc-en-ciel est attribué à la Vieille (cf. tableau précédent) et certains poissons labres, appelés « vieille», ont des écailles couleur arc-en-ciel. 
À l'issue de cette étude, on constate qu'en toponymie, et tout particulièrement en toponymie commémorative, il est très important d'adopter une approche pluridisciplinaire (littérature orale, archéologie, mythologie, lexicologie, etc.). L'exploitation de ces données diverses et variées permet de démontrer des concordances entre différentes aires linguistiques et culturelles. En effet, comme on a pu le voir, cette divinité ne se limite pas à l'aire celtique puisqu'on retrouve dans les aires germanique et romane - mais aussi slave et hellénique ${ }^{42}$ - des données ethnolinguistiques à son sujet. Le traitement de ces données permet également de révéler l'existence de figures divines ou légendaires qui sont la trace d'une mémoire collective à l'échelle de l'Europe. Certains mythes et légendes peuvent alors être qualifiés d'archétypes européens.

Concernant le motif "vieille », il correspond, selon mon hypothèse, à des vestiges culturels et linguistiques témoignant de la vénération d'une divinité appelée la Vieille. Contrairement à ce que peut laisser penser son nom, cette divinité est l'incarnation de la toute-puissance de la Nature considérée comme une grande déesse-mère nourricière, symbole de fécondité et de fertilité et maitresse du climat. Le terme « vieille », comme l'a rappelé Marie-Barbara Le Gonidec ${ }^{43}$, fait référence à la femme d'expérience, celle qui est source de savoirs notamment en matière de fécondité et de sexualité ${ }^{44}$.

Je tiens à souligner que la méthode adoptée ici se veut, avant tout, un outil de recherche transférable à d'autres figures culturelles emblématiques (telles que la Vierge, le diable ou Odin par exemple). En dressant des "portraits toponymiques » ${ }^{45}$, on pourra également savoir à

42. Comme j'ai pu le constater dans mes recherches.

43. Ethno-musicologue, chercheur associée au CRBC de Brest, lors de la journée « jeunes chercheurs » du Groupe de Recherche sur l'Économie Linguistique de la Bretagne (GRELB). Cette journée, organisée par Daniel Le Bris et Tanguy Solliec, s'est déroulée le 10 avril 2015, à la faculté des Lettres et Sciences Humaines de Brest, UBO/UBL.

44. C'est le cas en Galice (Xoán R. CubA, Antonio Reigosa \& Xosé Miranda, Diccionario dos seres miticos galegos, Espagne, Vigo, éd. Xerais de Galicia, 1999, p. 248, s.v. vella) et dans certaines localités de la Thrace (Iphigénie ANASTASSIADOU, « Deux cérémonies de travestissement en Thrace : le Jour de Babo et les Caloyeri », L'Homme, vol. 16, 1976, p. 69-101, éd. de l'EHESS).

45. S. GENDRON (2013: 14). 
quelle(s) réalité(s) géographique(s) elles sont le plus souvent associées. Ainsi, grâce à la base de données qui lui est consacrée, on constate que la Vieille désigne majoritairement des hauteurs, des rochers et des rivières, qu'elle est souvent associée à des paysages anthropomorphes ${ }^{46}$ et qu'elle est très souvent liée aux mégalithes.

\section{Bibliographie}

AkIN S., " Pour une typologie des processus redénominatifs », dans S. Akin (éd.), Noms et re-noms. La dénomination des personnes, des populations, des langues et des territoires, Rouen, Publications de l'Université de Rouen, 1999.

ALE (Atlas Linguarum Europae), Atlas linguistique européen, 2 vol., M. Alinei (dir.), Pays-Bas, Assen, Van Gorcum, 1983.

Alinei M. \& Benozzo F., «Les Celtes le long des côtes atlantiques : une présence ininterrompue depuis le Paléolithique», dans D. Le Bris (dir.), Aires linguistiques / Aires culturelles - Études de concordances en Europe occidentale: zones Manche et Atlantique, Brest, CRBC, 2012, p. 55-76.

Am Faclair Beag. An English-Scottish Gaelic dictionary incorporating Dwelly. Repéré à http://www.faclair.com/index.aspx

Belmont N., Paroles païennes : mythe et folklore : des frères Grimm à P. Saintyves, Paris, Imago, 1986.

Benozzo F., « Nomi totemici della balena in area celtica », Studi celtici, $\mathrm{n}^{\circ}$ 9, 2011, p. 15-25.

Repéré à http://www.continuitas.org/texts/benozzo_balena.pdf

BorLase W. C., The Dolmens of Ireland, 3 vol., London, Chapman \& Hall, 1897. Repéré à https://archive.org/details/dolmensofireland01borl

Contini M., «Temps météorologique et chronologique dans les proverbes sardes », Géolinguistique, n 13, 2012, p. 359-378.

DauZat A., La toponymie française, Paris, Payot, 1939.

Dauzat A. \& Rostaing Ch., Dictionnaire étymologique des noms de lieux en France, Paris, Larousse, 1963.

Evans D. S., An English and Welsh dictionary, vol. 2, pays de Galles, Denbigh, printed and published by Thomas Gee, 1858.

Repéré à : http://books.google.fr/books?id=RiMVAAAAYAAJ

46. Comme me l'ont confirmé certains informateurs écossais lors d'enquêtes de terrain sur les îles de Lewis et d'Islay. 
FalC'Hun F., Noms de lieux celtiques. $1^{\text {re }}$ série : Vallées et plaines, Rennes, éd. Armoricaines, 1966.

FAVEREAU F., Dictionnaire du breton contemporain, Dictionnaire breton-français, français-breton, Morlaix, Skol Vreizh, 1992.

Repéré à http://www.arkaevraz.net/dicobzh/index.php

FORBES A.R., Gaelic names of beasts (mammalia), birds, fishes, insects, reptiles, etc., Edinburgh, Oliver and Boyd, 1905.

Repéré à https: / / archive.org/details/gaelicnamesofbea00forb

Fossard J., «Lozère, royaume de la Vieille », Bulletin de la Société de Mythologie Française, $\mathrm{n}^{\circ} 251,2013$, p. 11-33.

Disponible en ligne à http:/ / www.lavieille.com/bsmf251.pdf

Frazer J. G., Le Ramean d'or - tome 3, Esprits des blés et des bois - Le bouc émissaire, Paris, Bouquins - Robert Laffont, 1983.

Fréminville (de) C., Antiquités de la Bretagne. Finistère, vol. 1, Brest, Lefournier et Deperiers, 1832.

GEndron S., Personnages illustres et noms de lieux. De Vercingétorix à Napoléon III, Arles, Errance, 2013.

Gendron S., Les noms de lieux en France - Essai de toponymie, Paris, Errance, 2008.

van GenneP A., Manuel de folklore français contemporain, Paris, Picard, 1947.

GPC (Geiriadur Prifysgol Cymru), Dictionnaire universitaire gallois.

Repéré à http://welsh-dictionary.ac.uk/gpc/gpc.html

GUÉNIN G., «Les rochers et les mégalithes de Bretagne. Légendes, traditions, superstitions ", Bulletin de la Société Académique de Brest, tome 35, 19101911, p. 191-280.

Hollocou P. \& Plourin J.-Y., De Quimperlé aux Montagnes Noires. Les noms de lieux et leur histoire entre Ellé et Isole, Emgleo Breiz, 2014.

Hull E., «Legends and Traditions of the Cailleach Bheara or Old Woman of Beare », Folklore, 38, n³, 1927, p. 225-254.

Repéré à http://www.jstor.org/stable/1256390

Joyce P. W., Irish Names of Places, 3 vol., Dublin, Phoenix Pub. Co., 1869, 1871, 1913. Repéré à https://archive.org/details/irishnamesofplac01joycuoft

LACroix J., Les noms d'origine gauloise. La Gaule des dieux, Paris, Errance, 2007.

MackenzIE D. A., Wonder Tales from Scottish Myth \& Legend, London, Blackie and Son Limited, 1917.

Repéré à https://archive.org/details/wondertalesfroms00mack

MED (Middle English Dictionary), The Regents of the University of Michigan.

Repéré à http://quod.lib.umich.edu/m/med/lookup.html 
Merceron J. E., La Vieille Carcas de Carcassonne. Florilège de l'bumour et de l'imaginaire des noms de lieux en France, Paris, Seuil, 2006.

Merriam-Webster, An Encyclopadia Britannica Company, dictionnaire unilingue anglais. Repéré à http:/ /www.merriam-webster.com/

Milio Carrín (de) C., La Creación del Mundo y otros Mitos Asturianos, Espagne, Oviedo, éd. de l'auteur, 2008. Repéré à www.academia.edu/7923667

Morgan T., The Place-Names of Wales, Newport, pays de Galles, Southall John E., 1912. Repéré à https:/ / archive.org/details/cu31924028086621

POLI M., «Quand la polysémie se généralise : le labre, le roitelet et l'euprocte se font "vieux". Les désignations relatives à la nature en langue corse ", La Bretagne Linguistique, $\mathrm{n}^{\circ}$ 19, Brest, CRBC, 2015, p. 27-42.

Rohlfs G., Lengua y cultura. Estudios linguisticos y folkloricos, Madrid, éd. Alcala, 1966.

SAINTYVES P., Corpus du folk:lore prébistorique en France, t. 3, Paris, Lib. Émile Nourry, 1936.

SÉBILlot P., Littérature orale de la Haute-Bretagne, Paris, Maisonneuve, 1881.

Soutou A., "Quelques fausses traces toponymiques du culte de Mercure », Revue Internationale d'Onomastique, XXI, 1969, p. 1-20.

Soutou A., «Toponymie, folklore et préhistoire : Vieille Morte », Revue Internationale d'Onomastique, 1954, p. 183-189.

StUART-GLenNiE J.S., Arthurian localities, Édimbourg, Edmonston and Douglas, 1869.

Tanguy B., Noms de lieux celtiques. Toponymie descriptive, Rennes, CRDP, 1975.

Watson W. J., Place-Names of Ross and Cromarty, Inverness, Écosse, The Northern Counties Print. and Pub. Co., 1904.

Repéré à https://archive.org/details/placenamesofross00watsuoft 